\title{
PRECISIONES LÉXICAS SOBRE LA INDUMENTARIA FEMENINA EN \\ EL SIGLO XVIII
}

\section{LEXICAL ACCURACY ABOUT WOMEN'S ATTIRE IN THE 18TH CENTURY}

\author{
Demelsa Ortiz Cruz \\ Universidad de Zaragoza \\ demelsa@unizar.es
}

\begin{abstract}
RESUMEN
El presente artículo, partiendo de los datos obtenidos en seis inventarios de bienes aragoneses del siglo XVIII (depositados en los fondos documentales del Archivo Histórico Provincial de Zaragoza), se centra en precisar algunos términos documentados en la centuria ilustrada propios de la indumentaria femenina de las clases altas aragonesas, en particular, vocablos referidos a adornos femeninos de la cabeza (el airón, la piocha y el tembleque), a joyas (los aguacates, las arracadas y broquelillos, la manillera y el reloj de repetición) y algunas voces alusivas a accesorios de ropa para el adorno femenino (en concreto, el excusalí, la falbalá y la palatina). Y todo ello queda ilustrado mediante retratos de la nobleza coetáneos a la documentación analizada.
\end{abstract}

\begin{abstract}
This paper is based on the data obtained in six inventories of Aragonese goods of the 18th century (placed in the Archivo Histórico Provincial de Zaragoza). It focuses on specifying some documented terms in the Enlightenment century, typical of the women's attire of the Aragonese upper classes, in particular, words referring to female head ornaments (airón, piocha and tembleque), jewels (aguacates, arracadas and broquelillos, manillera and reloj de repetición) and some voices alluding to feminine clothing accessories (specifically, excusali, falbala and palatina). All this is illustrated by portraits of the contemporary aristocracy to the analyzed documents.
\end{abstract}

Palabras clave: léxico, inventarios, siglo XVIII, Aragón, indumentaria femenina
Keywords: vocabulary, inventories, 18th century, Aragon, women's attire 


\section{INT'RODUCCIÓN}

Como es bien sabido, el análisis de relaciones de bienes, tipología documental estudiada tradicionalmente por los historiadores (Imízcoz, 1996; Santos, 2001, entre otros), es un objeto de especial interés desde un punto de vista lingüístico. Gracias a este tipo de investigaciones, relativamente recientes en el tiempo, se está pudiendo avanzar y precisar notablemente determinados aspectos de la evolución histórica de nuestra lengua, si bien, de modo particular, sobresalen los avances desde una perspectiva léxico-semántica a través de los cuales se está pudiendo matizar la configuración de la historia del léxico español.

En lo que concierne al siglo XVIII, el estudio léxico-semántico de estas fuentes documentales resulta todavía más próximo en el tiempo, ya que dicho periodo cronológico con demasiada frecuencia ha quedado desatendido por los historiadores de la lengua (idea apuntada por Calderón, 2012, p. 221 y también por Gómez Seibane y Ramírez Luengo, 2007, p. 11, entre otros). No obstante, desde época reciente, algunos estudiosos han resaltado la necesidad de incidir en el análisis de la documentación notarial de dicha centuria, tesoro del historiador de la lengua, ya que a través de ella se puede concretar la extensión geográfica y/o cronológica de un determinado vocablo, así como su naturaleza dialectal y diastrática (Puche, 2009a y 2009b sobre textos murcianos; Campos, 2012 sobre documentos de Galicia; Vivancos, 2012, sobre cuestiones morfológicas del murciano; Calderón, 2012, sobre aspectos gráficos y fonéticos de textos granadinos y americanos; Egido, 2008, 2012a, 2012b, 2012c y 2013 en documentación americana; y, en el caso de fuentes documentales dieciochescas de Aragón, Arnal, 2000 y, especialmente, los estudios de Ortiz, 2010, 2015a, 2015b, 2017a, 2017b, 2018 y en prensa).

Por consiguiente, en el presente estudio vamos a partir de los datos obtenidos en algunas relaciones de bienes aragonesas del siglo XVIII que se encuentran depositadas en los fondos documentales del Archivo Histórico Provincial de Zaragoza (AHPZ), trabajo que conecta con otro de mayor envergadura (Ortiz, en prensa) ${ }^{1}$. Más

\footnotetext{
${ }^{1}$ Este estudio se desprende de nuestra Tesis doctoral titulada Estudio léxico-semántico de inventarios de bienes

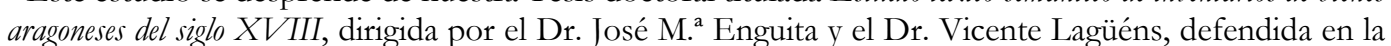
Universidad de Zaragoza en el mes de diciembre de 2015, con la que se obtuvo la calificación de
} 
concretamente, se trata de una selección de seis inventarios de bienes (citados y referenciados en el apartado final de $\int$ Fuentes primarias) procedentes de las secciones de las casas nobles aragonesas de Aranda-Hijar y Argillo, así como de la sección de Pleitos Civiles de Casas Nobles de dicho Archivo, por lo que nos centraremos en precisar algunos términos documentados en la centuria ilustrada propios de la indumentaria femenina de las clases altas aragonesas, en particular, vocablos referidos a adornos femeninos de la cabeza (el airón, la piocha y el tembleque), a joyas (los aguacates, las arracadas y broquelillos, la manillera y el reloj de repetición) y algunas voces alusivas a accesorios de ropa para el adorno femenino (en concreto, el excusalí, el falbalá y la palatina $)^{2}$.

La elección de dichos campos onomasiológicos radica en que son estos los que atestiguan una mayor cantidad de voces, pues las mujeres son, las más de las veces, quienes más cuidan de su aspecto personal, de ahí que proliferen aderezos de diversa índole (con la consiguiente denominación al respecto) para cubrir estas necesidades. Por otro lado, las palabras aquí referidas se han seleccionado, como se verá en cada una de las explicaciones, por su interés semántico, regional o cronológico, puesto que se da cuenta de nuevos datos documentales que permiten precisarlas. Para ello, se ha llevado a cabo una revisión bibliográfica sobre los términos acopiados, a través de la consulta de las diferentes ediciones del Diccionario académico (con especial interés a la información contenida en el llamado Diccionario de Autoridades, elaborado entre 1726 y 1739, coetáneo a nuestros textos), junto a otros diccionarios generales (como el Diccionario castellano con las voces de ciencias y artes y sus correspondientes en las tres lenguas francesa, latina e italiana, de Esteban de Terreros y Pando, del año 1787, también del siglo XVIII, entre otros). Además, para completar el estudio con datos de carácter cronológico, se ha recurrido al Diccionario crítico etimológico castellano e bispánico (DECH), de Juan

Sobresaliente Cum Laude. Aprovechamos esta ocasión para hacerles público nuestro agradecimiento por sus sabios consejos durante el periodo de realización de nuestro trabajo doctoral.

2 Para una revisión completa de la evolución de la moda en el siglo XVIII, véase el artículo de Leira (2007) y para el caso del calzado, el de García (2006). Si se quiere ahondar en los diferentes estilos de moda en diversas épocas del siglo ilustrado, puede consultarse para la robe à la française (la bata) el estudio de Benito (2006); para la robe à la polonaise (la polonesa), el de Redondo (2007); para la robe à l'anglaise (el vaquero), el de Leira (2008); el traje de maja, el de Gómez del Val (2008); y para la camisa neoclásica, el de Bermejo (2016). 
Corominas y José Antonio Pascual (1980-1991), así como a la consulta del Corpus diacrónico del español (CORDE) de la Real Academia Española, entre otras fuentes y bases de datos.

A continuación, se presenta el estudio de cada una de las voces enumeradas ${ }^{3}$ :

\section{PRECISIONES LÉXICAS SOBRE INDUMENTARIA FEMENINA DEL SIGLO XVIII}

\subsection{ADORNOS DE LA CABEZA \\ 2.1.1. Airón}

Las mujeres en el siglo XVIII, al igual que ahora, acostumbraban a adornar su pelo con diferentes tipos de adorno a un lado de la cabeza, como flores, lazos o joyas. Dependiendo de su constitución y forma, estos adornos recibían diferentes denominaciones para precisarlos. Entre ellos, se encontraba el airón, que es un "adorno de plumas, o de algo que las imite, en cascos, sombreros, gorras, etc., o en el tocado de las mujeres" (DLE, s.v.).

Aunque es una voz antigua en el idioma (documentada, de acuerdo con los datos manejados, desde finales del siglo XV con el significado anotado $)^{4}$, al menos en el siglo XVIII había ido recibiendo diferentes usos: así, según consta en el Diccionario de Autoridades (NTLLE, 1726-1739, s.v.), este adorno era tradicionalmente de color negro ("cierta cantidad de plumas negras de diferentes aves, de que se formaba un penacho", de acuerdo con el Autoridades) y antiguamente se denominaba martinete, "por ser lo regular hacerse de las plumas de un ave llamada así”. Se advierte en este repertorio lexicográfico que "Covarrubias dice que es lo mismo que garæóta, en que padece notable equivocación", pues ambos se diferencian por su color: "Porque la garøóta es el

\footnotetext{
${ }^{3}$ Los ejemplos que a continuación se citan procedentes de nuestros inventarios se transcriben siguiendo el principio de la mayor fidelidad posible al manuscrito original en lo ateniente a la diferencia entre ciertas variantes gráficas, si bien, con el objeto de facilitar la lectura del texto, hemos actualizado algunas cuestiones de acuerdo con las normas ortográficas actuales, como es el uso de mayúsculas y minúsculas, la puntuación o el empleo de la tilde.

${ }^{4}$ El primer registro del CORDE es de $c$. 1492: “Tornándolo a tañer, salyeron en cada vanco tres damas hermosas, vestidas todos de una manera, del brocado y tercyopelo carmesy de las vandas, con unos sonbreretes de lo mysmo y en cada uno un penachón de ayrón muy grandes y muy blancos" (Anónimo, La corónica de Adramón, c. 1492, CORDE).
} 
plumage que la garza tiene en la cabeza, el qual es blanco; y aunque dél se usaba como del airón, hai la diferencia que queda dicha" (figura 1$)^{5}$.

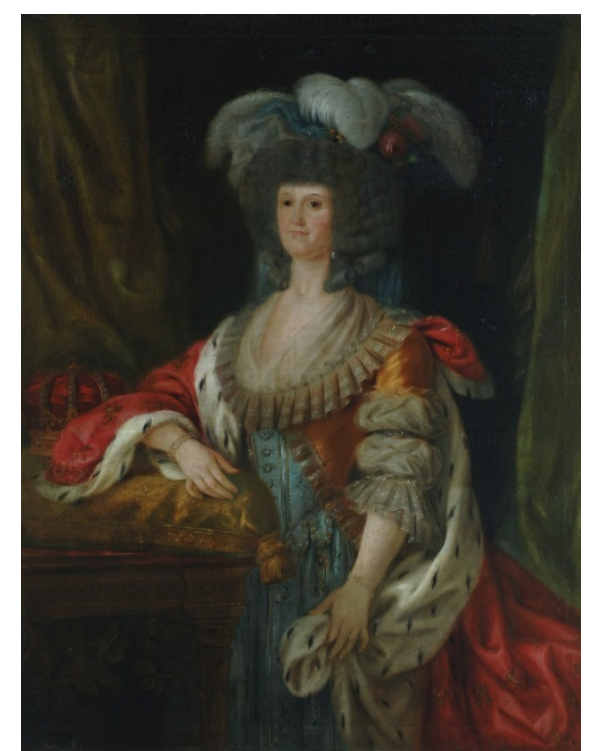

FIGURA 1. Ejemplo de garzota de plumas blancas en el retrato de $M \cdot{ }^{a}$ Luisa de Parma, atribuido a Antonio Carnicero (c. 1789).

Además del uso decorativo en gorras, sombreros y morriones, interesa destacar que "usaban también las mujeres, ponienselos en sus tocados; las que no solo le trahían de plumas, sino también imitado de piedras preciósas" (NTLLE, 1726-1739, s.v.). Es decir, que el airón podía estar constituido tanto de plumas (a la manera tradicional) como de joyas, cuestión que también queda recogida en la definición de ayrón en el Diccionario académico de 1770: "Llámase hoy ayron el penacho pequeño de una ó mas plumas (comunmente negras) de esta ú otras aves, de que usan las mugeres por adorno en la cabeza: y por semejanza el que se hace de plata ú oro con piedras preciosas, ó bien de hilos de vidrio ú otra materia, imitando la figura de las mismas plumas" (NTLLE, 1770, s.v.). A juzgar por la documentación manejada, la denominación de airón de pedrería

\footnotetext{
${ }^{5}$ La equivocación por el color entre la gazorta y el airón se atestigua desde antaño: obsérvese, por ejemplo, el anterior ejemplo del CORDE donde se habla de "unos sonbreretes de lo mysmo y en cada uno un penachón de ayrón muy grandes y muy blancos".

Un ejemplo de garzota lo podemos observar en el retrato de M. ${ }^{a}$ Luisa de Parma, la esposa de Carlos IV, atribuido a Antonio Carnicero, que fue pintado hacia 1789 (figura 1).

Para la selección de las obras pictóricas presente en este artículo, se ha contado con la inestimable ayuda del prestigioso experto en Historia del Arte del siglo XVIII, Javier Martínez Molina, a quien agradecemos encarecidamente su ayuda para ilustrar los términos reseñados con las citadas obras de arte.
} 
se atestigua ya a principios del siglo XVII ${ }^{6}$. Un ejemplo de los inventarios de bienes analizados con este sentido es el de (1):

(1) Quatro pares de botones de diamantes. Vn ayrón o piocha de diamantes. Las manillas de aljófar. El rejox de oro (p. 4, línea 26) 7.

Obsérvese que en este caso (1) el ayrón aparece inventariado junto a otras joyas, como los "botones de diamantes", las "manillas de aljófar" (esto es, una pulsera compuesta por varias vueltas, como explicaremos más adelante en el caso de manillera; vid. \$2.2.3.) y el "relox de ôro", de ahí que no pueda dudarse el hecho de que se trate, pues, de un airón de pedrería ( $\mathrm{y}$ no de plumas, a la manera tradicional). Además, en el ejemplo aludido, ayrón se hace equivalente mediante la conjunción "o", de carácter explicativo en nuestra relación de bienes, a la piocha, nuevamente otra joya cuya incorporación a nuestra lengua resulta más moderna en el tiempo, como veremos a continuación (vid. \2.1.2.).

Una representación del airón en un cuadro de la época lo tenemos presente en el retrato de M. ${ }^{a}$ Teresa de Vallabriga, esposa del infante don Luis de Borbón (hermano del rey Carlos III), pintura del ilustre Francisco de Goya, quien la retrató en el año 1783 con un airón de plumas negras adornado a su vez por uno de pedrería (figura 2).

\footnotetext{
6 "En los cinco ayrones grandes, engastados treinta y cinco diamantes, los treinta prolongados, tablas, unos más largos que otros, y los cinco de los remates, lanzetas" (Anónimo, Inventarios reales. Bienes muebles que pertenecieron a Felipe II, c. 1600, CORDE).

${ }^{7}$ Reparto de los bienes que quedaron por la muerte de Isabel López. de Texeda, condesa de Morata, marquesa de Villaverde. Sin fecha. Consta de 9 páginas, sin numerar. Signatura: AHPZ, Argillo 131-6.
} 


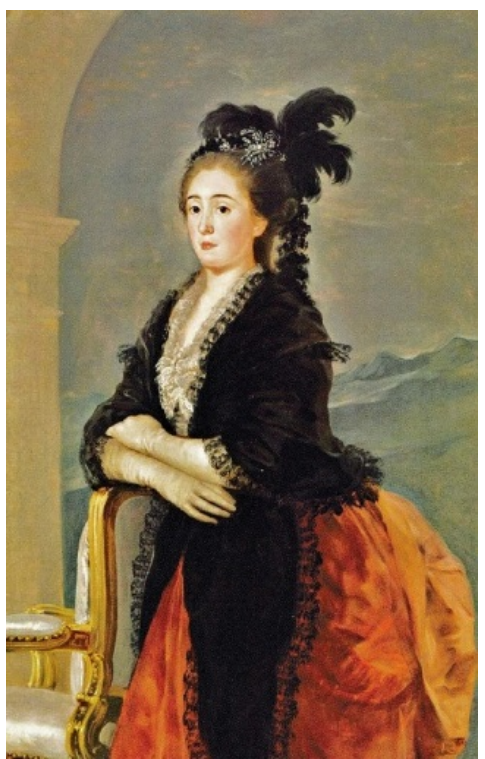

FIgurA 2. Ejemplo de airón en el retrato de $M .^{a}$ Teresa de Vallabriga, de Francisco de Goya (1783).

\subsubsection{Piocha}

Otro adorno más lujoso para el pelo era la piocha, esto es, la “joya de varias formas que usaban las mujeres para adorno de la cabeza" (DLE, s.v.). Dicha palabra proviene del italiano pioggia 'lluvia', quizá “por alusión a la lluvia de oro de Júpiter” (DECH, s.v. lluvia). Con el significado anotado, aparece por primera vez, de acuerdo con los datos del CORDE, en un texto del año $1761^{8}$, tan solo tres años anterior al de nuestro manuscrito (2) de 1764. Por lo tanto, parece tratarse de una voz escasamente documentada en fechas anteriores al siglo XVIII.

\footnotetext{
8 "Lista de lo que necesita una dama de moda para equiparse antes de darse al público: Primeramente: el peinado, las flores, las piochas, la cotilla, lavatorio de manos y cara. El alfilerón para el despunte; el vaquero; la bata ó casaca; corbata ó pañuelo, y á mano el acerico para el plegatorio general. El ramo si es día de él ó le hubiere; los pendientes; el sofocante ó collar; el brial. Si no bata ó vaquero, la basquiña; las sortijas; los guantes ó pitones; los brazaletes; los vuelos, no olvidando la cofieta, manteleta ó capotón. Si es invierno, nada; si es verano, el manto ó la mantilla; el alfiler para prenderla en el occipucio; el manguito. Si es tiempo, el abanico; la caja de barro; vinagrillo; palillo ó tabaco común; el reloj, y la que no le tenga, póngasele con el deseo; pañuelo blanco; el de narices (por no decir de mocos); si hay, los dos; las chinelas y el coche (la que le tuviere); el paje, ó irse sola, ó agarrarse de alguno; un libro para sacarle alguna vez, aunque sea la Guia de forasteros. No se pone más porque no es de aquí" (R. de la Cruz, El pueblo sin mozas, 1761, CORDE).
} 
(2) En vna cagita de zapa, vna mariposa o piocha de diamantes brillantes y rubíes con su abuja de plata. En otra caxita de lo mismo, vna piocha de plata, guarnecida de diamantes y esmeraldas (p. 64, líneas 16-19)9 .

Desde un punto de vista lexicográfico, el primer diccionario en el que queda recogida la palabra piocha es el de Terreros y Pando (1987 [1786-1793], s.v.), con el sentido de “joya o especie de flor con pedrería o sin ella, que se ponen las mujeres en la cabeza sobre la frente o a un lado". Por su parte, la Academia espera a la 4. ${ }^{\text {a }}$ edición de su Diccionario (NTLLE, 1803, s.v.) para incluirla, con una definición menos detallada que la anterior: "Joya de varias figuras de que usan las mugeres para adorno de la cabeza", acepción que se mantiene sin cambio alguno hasta la actual edición del DLE (s.v.).

Una piocha con forma de mariposa, parecida a la inventariada en nuestro documento, la podemos ver expuesta en la cabeza de la mujer retratada de Tomasa de Aliaga, viuda de Salcedo, realizado por Antonio Carnicero, hacia 1787 (figura 3).

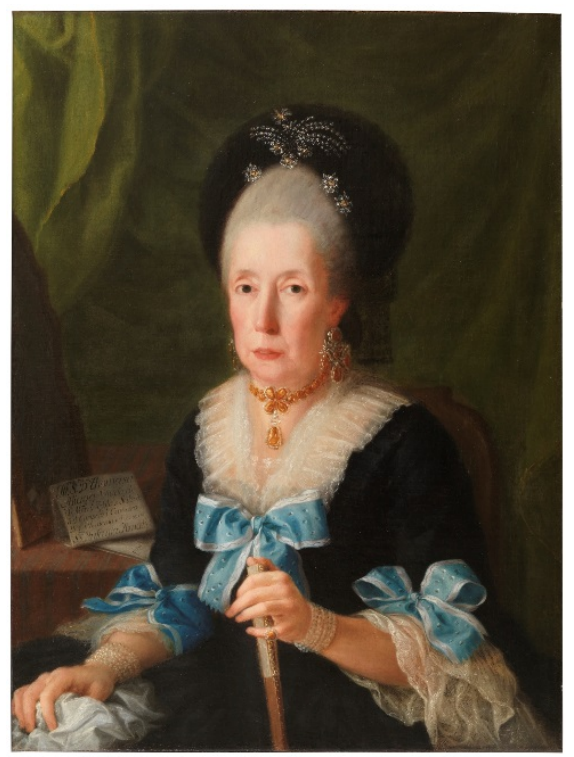

FIGURA 3. Ejemplo de piocha con forma de mariposa en el retrato de Tomasa de Aliaga, viuda de Salcedo, de Antonio Carnicero (c.1787)

\footnotetext{
${ }^{9}$ Inventario de bienes y objetos encontrados en unas casas, sitas en la calle del Coso de Zaragoza, que pertenecieron a D. ${ }^{a}$ Prudenciana Portocarrero Funes de Villalpando, duquesa de Hijar. Fechado en Zaragoza, a 9 de junio de 1764. Consta de 75 páginas, sin numerar. Signatura: AHPZ, ADH, I-357-31/2.
} 


\subsubsection{Tembleque}

Un último adorno para el pelo al que nos vamos a referir es el del tembleque, el cual, según se explica en el Diccionario de Autoridades (NTLLE, 1726-1739, s.v.), es un "adorno que usan las mujeres para la cabeza que es una flor o botón de diamantes, u otras piedras, preso a una aguja de plata u oro, que por estar retorcido tiembla con el pelo". Dicho vocablo figura en estos dos documentos aragoneses dieciochescos (3 y 4):

(3) La joya en forma de alamar con pendientes y cruz correspondientes, con siete mariposas o tembleques (p. 1, línea 8) ${ }^{10}$.

(4) Vna joya en forma de alamar con pendientes y cruz correspondientes con siete tembleques o mariposas de oro y plata, diamantes y esmeraldas (p. 5 , línea 16$)^{11}$.

Tembleque es un derivado de temblar, procedente del latín vulgar TRĔMŬLĀRE íd., derivado de TRĔMŬLUM ‘tembloroso' y este de TRĔMĔRE 'temblar' (DECH, s.v. temblar). Con el sentido anotado, se documenta desde la inclusión de tembleque en el Diccionario de Autoridades (NTLLE, 1726-1739, s.v.); el primer registro que actualmente figura en el CORDE es unas décadas posterior a este repertorio $^{12}$, por lo que se trata de una voz escasamente documentada en época anterior al siglo XVIII.

Si comparamos los ejemplos aducidos para la piocha y el tembleque, podemos aventurar que el primero de estos términos quizá pueda considerarse de un significado más genérico, de ahí que una de las características propias y específicas del segundo, el de estar "preso a una aguja", no se especifique en los ejemplos documentados de (3) y (4), mientras que sí en el de (2). No obstante, la caracterización de ambos vocablos con el sentido de joya no debía de estar muy bien delimitado: obsérvese que, al menos en el caso de la piocha, en su entrada no se especifica la forma que le es propia (“joya de varias formas") y poco más se dice en el de tembleque ("es una flor o botón de diamantes, u otras piedras"); por otro lado, la modernidad en la adopción de ambas voces podría justificar el hecho de que en los ejemplos

10 Tasaciones de los bienes más valiosos de D. ${ }^{a}$ María Agustina Clavero y Dara. Incluye cinco tasaciones, fechadas en Zaragoza, a 24 de octubre, y a 2, 3 y 4 de noviembre de 1732 (la última no tiene fecha). Consta de 5 páginas, sin numerar. Signatura: AHPZ, J-302-1.

11 Inventario de los bienes pertenecientes a la casa del Sr. Segovia. Fechado en Zaragoza, a 26 de marzo de 1733. Consta de 7 páginas, sin numerar. Signatura: AHPZ, J-302-1.

12 "En la parte anterior y superior ponen varios tembleques de diamantes y, con el mismo cabello, hacen unos pequeños rizos que, siguiendo su ceja encaracolados, baxan de la parte superior de las sienes hasta la medianía de las orejas, como que salen naturalmente del mismo pelo, y ponen dos parches de terciopelo negro algo grandes en las sienes, á la manera de los que se han dicho en otras descripciones que igualmente las agracian" (A. de Ulloa, Viaje al reino del Perú, 1748, CORDE). 
seleccionados aparezcan tanto piocha como tembleque como voces sinónimas a la "mariposa" (revísense los citados ejemplos 2, 3 y 4). Aun con todo, el término tembleque parece venir motivado por el mayor movimiento de las piezas de joyería que lo componen, mientras que quizá la piocha quedara más fijada al cabello, impidiendo su movimiento o que este se viera notablemente reducido.

Un cuadro ilustrativo de lo que podría ser un tembleque en forma de flor se puede observar en el retrato de Bárbara de Braganza, la esposa de Fernando VI, del pintor francés Jean Ranc, pintado hacia 1729 (figura 4).

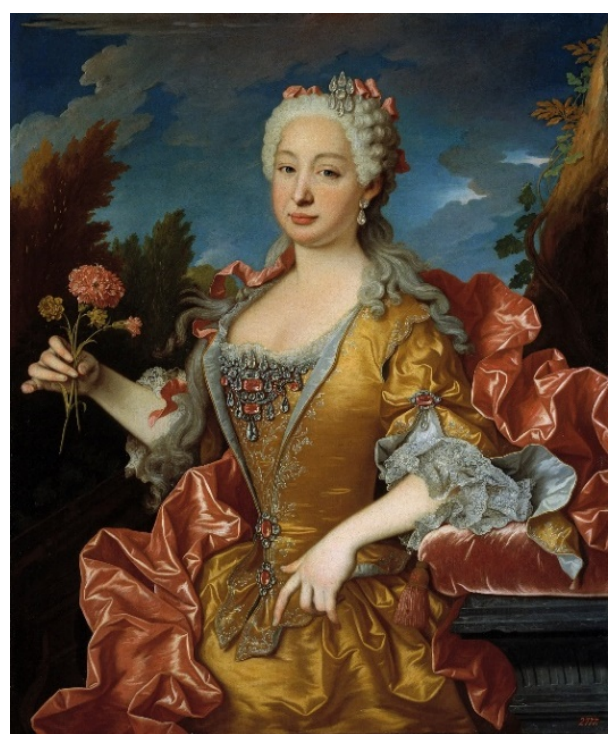

FIGURA 4. Ejemplo de tembleque en forma de flor en el retrato de Bárbara de Braganza, de Jean Ranc (c. 1729).

\subsection{JOYAS}

\subsubsection{Agnacates}

Con el término aguacate se venía a designar a la "esmeralda de forma de perilla" (DLE, s.v.), palabra que proviene del náhuatl awákatlíd. (DECH, s.v.). Aunque con el significado de fruto tropical aparece ya en el padre Las Casas (hacia 1560), con el sentido presente en los registros de nuestros inventarios de bienes, se halla al menos desde 1646 (DHLE, s.v.). En el ejemplo presente en nuestros inventarios (5) se puede comprobar que al citar el término estudiado se matiza que se trata de una piedra preciosa y no de un fruto tropical:

(5) Vnos anillos con dos aguacates de esmeraldas (p. 1, línea 14) ${ }^{13}$.

13 Vid. nota 10. 
En el Diccionario de Autoridades (NTLLE, 1726-1739, s.v.) aguacate figura ya con la acepción de 'esmeralda', donde se matiza que de esta "solo se diferéncia en que no es tan perfecta, y en que es de hechúra redonda, ò prolongáda". También se lematiza en el repertorio de Terreros y Pando (1987 [1786-1793], s.v.). Asimismo esta acepción consta en el DUE (s.v.) y en la actual edición del DLE (s.v.).

Un retrato en el que se puede observar, no un anillo (como en el ejemplo procedente de nuestros inventarios de bienes), sino unos pendientes y un collar formados por aguacates de esmeralda es el de Paula Melzi de Eril, marquesa de Lazán (y madre del general José de Palafox), pintado por Francisco Bayeu entre 1770 y 1775 (figura 5).

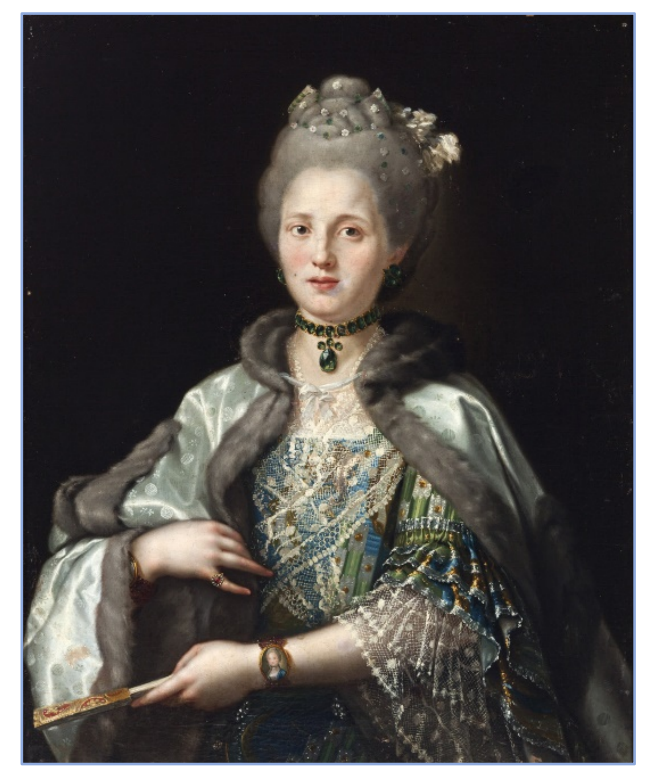

FIGURA 5. Ejemplo de aguacate en un collar en el retrato de Paula Melzi de Eril, marquesa de Lazán, de Francisco Bayeu (c. 1770-1775).

\subsubsection{Arracadas y broquelillos}

Hablando de pendientes, encontramos en la documentación dieciochesca voces como las de arracadas y broquelillos. Primeramente, la arracada, palabra antigua en el idioma, se define desde la 1. a edición del Diccionario académico (NTLLE, 1780, s.v.) como "qualquiera de los dos pendientes que se ponen las mugeres en las orejas, por gala o adorno" (6).

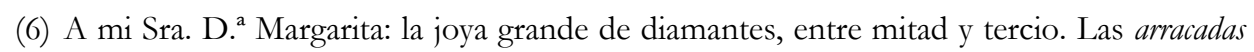
con quatro diamantes grandes (p. 2, línea 18) ${ }^{14}$.

${ }^{14}$ Vid. nota 7. 
Junto a esta, aparece el vocablo broquelillo (7), concebido como "botón pequeño, con colgante o sin él, que, pendiente de las orejas, usan las mujeres como adorno”, término en desuso según la actual edición del DLE (s.v.):

(7) Dos broquelillos con piedras adiamantadas en plata. [...] Dos pares de broquelillos de ensaladilla en plata (p. 66, líneas 1 y 13) 15 .

Se trata de un derivado de broquel, procedente del francés antiguo bocler (hoy bouclier), este de bocle "guarnición de metal que el escudo llevaba en su centro" y este del latín BŬCCǓLAM íd., diminutivo de BUCCAM 'mejilla' (DECH: s.v. broquel). Aunque broquelillo no se considera en el DECH, hallamos noticias de esta voz desde el primer tercio del siglo XVII, de acuerdo con los datos del CORDE ${ }^{16}$. En cuanto a su consideración como entrada lexicográfica, se incorpora por primera vez en el Diccionario de Autoridades (NTLLE, 1726-1739, s.v.), donde se define como "botoncillo de que está pendiente la piedra, almendra, ò arracadas, con que adornan las oréjas las mugéres”. Obsérvese bien que en esta acepción se especifica que la arracada es un elemento que se añade al broquelillo y no un tipo especial de pendiente, sentido más acorde a la documentación analizada, pues las arracadas figuran de manera independiente, como un objeto con entidad propia, como se puede observar en el citado ejemplo de (6).

En los retratos femeninos a los que hemos aludido en las secciones anteriores podemos ver representados diferentes tipos de arracadas y broquelillos: revísese de nuevo el de Tomasa de Aliaga (figura 3) con grandes arracadas y los de Bárbara de Braganza (figura 4) y Paula Melzi de Eril (figura 5) con broquelillos (los últimos compuestos, como ya hemos visto, de aguacates de esmeralda).

\subsubsection{Manillera}

Otro nombre interesante de joya es la manillera (8), derivado morfológico de manilla, este último definido en el Diccionario de Autoridades como "el adorno que trahen las

${ }^{15}$ Vid. nota 9. Recuérdese que la ensaladilla es "un joyél compuesto y matizado de diferentes piedras preciosas, como diamantes, rubies, esmeraldas, etc.", siendo el joyel la "joya pequeña, que à veces no tiene piedras", según las correspondientes entradas del Diccionario de Autoridades (NTLLE, 1726-1739, s.vv.).

16 "Mas treynta y tres mill y ochozientos sesenta y quatro maravedis en que se taso unas arracadas de perlas y broquelillos" (Anónimo, Carta de dote y arras entre don Luis de Sotomayor y doña Beatriz Belarde, 1629, CORDE). 
mugéres en las muñecas, compuesto de unas sartas que dán varias vueltas, de perlas, coráles, granátes ù otras cuentas" (NTLLE, 1726-1739, s.v.).

(8) A mi Sra. D. ${ }^{a}$ M. ${ }^{a}$ Francisca: vna cruz de diamantes en plata. Las dos arracadas en plata. Dos manilleras de diamantes en plata. El oro de dos sortijas de piedras falsas (p. 3, línea $21)^{17}$.

Manillera es, pues, un derivado de manilla, y este de mano, del latín MANUM íd. (DECH: s.v. mano), si bien este término sufijado no se recoge en el DECH, ni figura en ningún diccionario (tanto preacadémico como académico) ni se registran casos en el CORDE ni en el CorLexIn, por lo que podría tratarse de una forma alternante con manilla con el sentido anotado, escasamente documentada en el siglo XVIII, a juzgar por los datos a los que se ha tenido acceso: en efecto, el ejemplo atestiguado no deja lugar a dudas de que se trata de un tipo especial de joya, puesto que en el contexto que le precede y le sigue aparecen nombres de joyería, como la "cruz de diamantes", las "arracadas en plata" y las "sortijas de piedras falsas".

Un ejemplo muy claro de manillas o manilleras lo podemos ilustrar con el retrato, de autor desconocido, de Mariana de Zuasti (c. 1765), mujer murciana de clase alta quien las luce en sendas muñecas (figura 6).

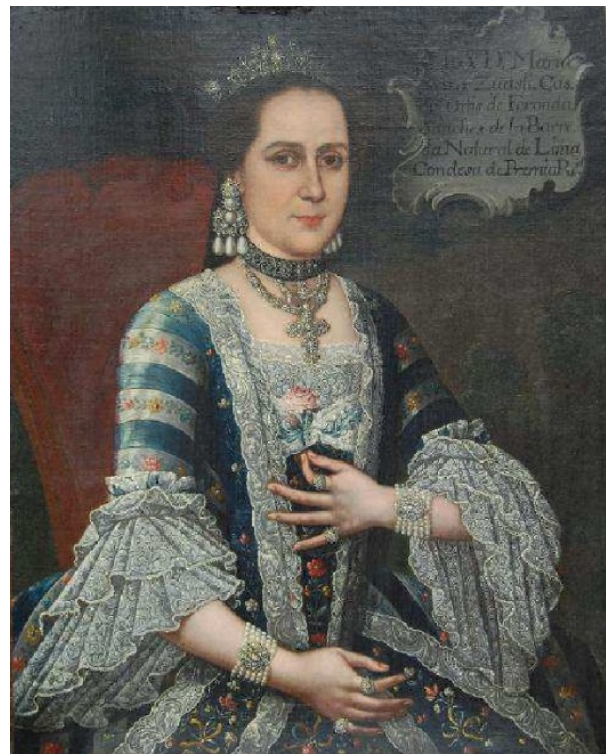

FIGURA 6. Ejemplo de manilleras en ambas muñecas en el retrato de Mariana de Zuasti, de autor murciano desconocido (c. 1765)

17 Vid. nota 7. 


\subsubsection{Reloj de repetición}

Con la expresión compuesta reloj de repetición se alude a aquel tipo de reloj que "da la hora siempre que se aprieta un muelle", según queda explicado en la 4. ${ }^{a}$ edición del Diccionario de la Academia (NTLLE, 1803, s.v.). En dos inventarios de bienes aragoneses se localiza este objeto (9) y (10):

(9) Dos reloxes de charol de repitición (p. 8, línea 15) ${ }^{18}$.

(10)Dos reloxes de oro de repetición, ambos con cadena de oro, el vno más grande que el otro; el mayor con caxa de zapa verde y en la cadena, vna cagita de piedra blanca guarnecida de oro; y en el más pequeño, vn camafeo guarnecido de chispas de diamantes (p. 71, línea 10) ${ }^{19}$.

Reloj de repetición está formado por reloj (vOz griega tomada del latín HOROLŎGIUM 'reloj de sol', 'reloj de arena', a través del catalán antiguo y dialectal relotge, hoy rellotge; $D E C H$, s.v. reloj), la preposición de y el sustantivo repetición (relacionado con el verbo repetir, del latín REPĚTĔRE íd.; DECH, s.v. pedir).

Los relojes de repetición son un invento de finales del siglo XVII: fue el inglés Quare quien introdujo por primera vez entre 1680 y 1686 la repetición de horas y cuartos en un reloj de bolsillo a través de un timbre situado en el fondo de la caja (Landes, 2007: 262). De este modo, la expresión reloj de repetición comienza a documentarse en los textos españoles desde el siglo XVIII; prueba de ello son nuestros registros procedentes de inventarios de los años 1729 (9) y de 1764 (10), y los ejemplos del CORDE, de mediados de dicha centuria ${ }^{20}$. Por otro lado, esta expresión se recoge, como ya ha quedado apuntada, desde la 4. edición del Diccionario académico (NTLLE, 1803, s.v.), con el significado anotado, y se mantiene hasta la actual edición del DLE (s.v.), con una ligera variación significativa: "Reloj que repite la señal horaria que acaba de dar". Por lo tanto, nos hallamos ante un nuevo caso de una expresión escasamente documentada en fechas anteriores al siglo XVIII.

${ }^{18}$ Inventario del menaje de la casa del conde de Aranda en Zaragoza. Fechado en Zaragoza, en noviembre de 1729. Consta de 13 páginas, sin numerar. Signatura: AHPZ, ADH, III-74-4.

19 Vid. nota 9.

20 «Marqués. (Dándole un reloj de repetición.) / Esto es cosa más pesada. / Ocioso. Venga, y no olvidéis lo que esta / repetición os señala: / pues agentes y relojes / son tan críticas alhajas / que, si no se les da cuerda / todos los días, se paran» (Ramón de la Cruz, El agente de sus negocios, 1762, CORDE). 
Francisco Bayeu retrató hacia 1765-1767 a la condesa de Estepa, Mariana de Urriés y Pignatelli, donde se la puede ver con unos relojes de repetición de cadena colgados de la cintura (figura 7).

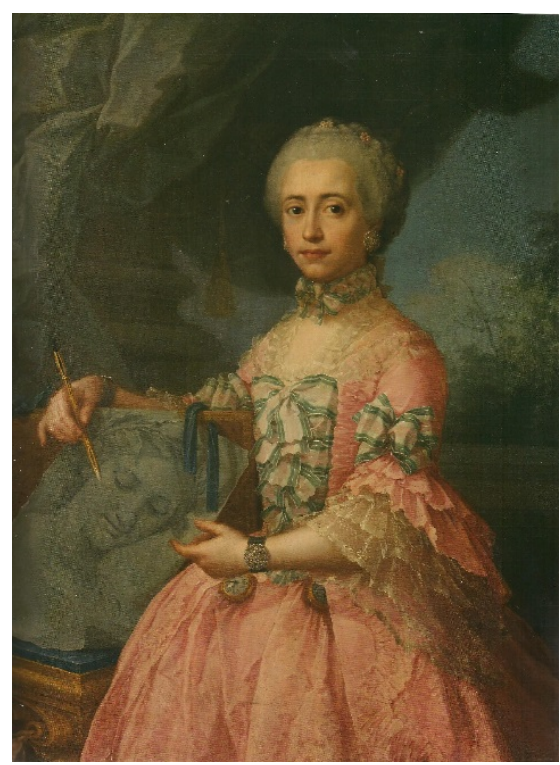

FIGURA 7. Ejemplo de relojes de repetición en el retrato de Mariana de Urriés y Pignatelli, de Francisco Bayeu (c. 1765-1767).

\subsection{ACCESORIOS}

\subsubsection{Excusalí}

Con el término excusali (11) nos referimos a un "delantal pequeño" (DLE: s.v.), voz procedente del italiano septentrional scossal 'delantal', el cual a su vez deriva de scos 'regazo', descendiente del longobardo (esto es, el dialecto del alto alemán hablado en la Italia medieval) shanzíd., que fue transmitido probablemente por vía de una forma diminutiva occitana *escoussali(n) (DECH, s.v. excusali).

(11)Más 8 onzas y tres quartas de dichos [adarmes] con encajes de oro anchos y angostos para guarnizión de escusali, paletina y petos, que se entregaron a la bordadora (p. 2, línea $20)^{21}$.

La variante escusalín, que alternó en un primer momento con la anotada, se incorpora en el Diccionario de Autoridades (NTLLE, 1726-1739, s.v.), donde se indica que es "voz francésa, nuevamente introducida", y se mantuvo en las dos primeras ediciones del

${ }^{21}$ Memoria de los vestidos de boda de D. ${ }^{a}$ Ana M. ${ }^{a}$ del Pilar Silva y Portocarrero, con sus hechuras y precios. Fechado en el año 1739. Consta de 6 páginas, sin numerar. Signatura: AHPZ, ADH, I-375-1/4. 
Diccionario académico (1780 y 1783), así como también en el repertorio de Terreros y Pando (1987 [1786-1793]: s.v.). Este último autor comenta que "comunmente se dice escusali". En cuanto a escusalí, se incluye desde la 3. ${ }^{a}$ edición del Diccionario de la Academia (NTLLE, 1791, con el sentido de 'devantal') hasta la actual edición del DLE, en la que se remite directamente a excusalí. Por último, excusalí también se considera como entrada académica desde la 3. a edición (NTLLE, 1791, como “delantal pequeño”). Observamos, por tanto, que la importancia de este registro documental radica en que se halla escasamente atestiguado en el siglo XVIII, a juzgar por las fuentes manejadas ${ }^{22}$.

El excusalí lo lleva, por ejemplo, María Bayeu, hermana de los pintores Francisco Bayeu, Ramón Bayeu y Manuel Bayeu, retratada por el primero de sus hermanos en el año 1783 (figura 8).

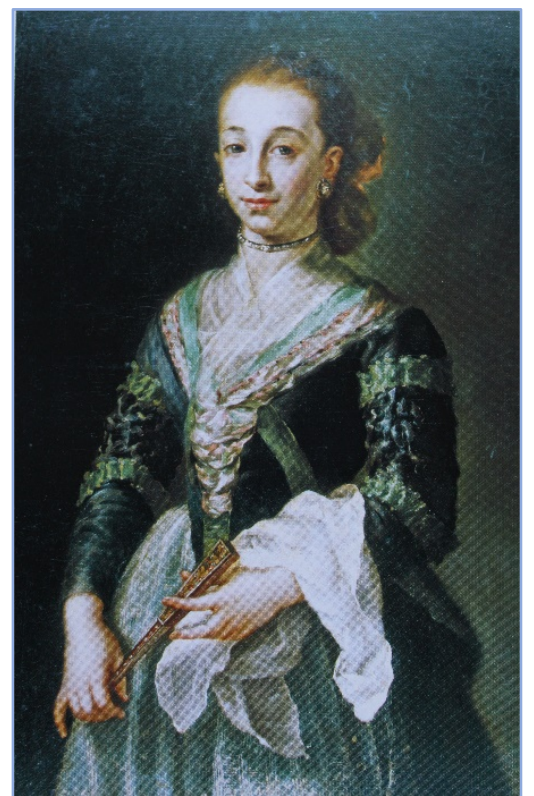

FIgURA 8. Ejemplo de excusali encima de la falda en el retrato de María Bayeu, de Francisco Bayeu (1783).

\subsubsection{Falbalá}

En cuanto al falbalá, se trata, según se especifica en el Diccionario de Autoridades (NTLLE, 1726-1739, s.v.), de un “adorno compuesto de una tira de tafetán o de otra tela, que rodea las basquiñas y briales de las mujeres, toda alechugada y cosida por el canto

22 De hecho, el primer registro del CORDE, anterior al aquí mencionado, pertenece a Moratín: "Si una bata entonces saco, / sacaré otra para ti, / un reloj y excusali / con su caja de tabaco" (N. Fernández de Moratín, La petimetra, 1762, CORDE). 
superior y suelta por el inferior”, adorno que también se utilizaba en el tontillo, esto es, "una especie de faldellín, ò guardapies, que usan las mugeres, con aros de vallena, ù de otra materia, puestos à trechos, para que ahueque la demás ropa”, a juzgar por el ejemplo atestiguado en nuestros inventarios (12):

(12) Más 22 varas de tafetán doble encarnado para tontillo guarnezido con farbalaes de lo mismo (p. 19, línea 9)23.

Falbalá es un término que proviene del francés falbala íd., adaptación del lionés farbéla 'franja' que parece ser una "voz de creación expresiva” (DECH, s.v.). A juzgar por las fuentes manejadas, se trata de una voz escasamente documentada en el siglo XVIII, pues el primer registro es su inclusión en el Diccionario de Autoridades (NTLLE, 17261739, s.v.) con el significado anotado ${ }^{24}$, información a la que se añade que "el uso de este adorno es moderno en España”; se dice en este repertorio, además, que la variante farbalá, que es la localizada en nuestros inventarios, es la común en la época. Desde la 3. ${ }^{a}$ edición del Diccionario académico (NTLLE, 1791, s.v.) hasta la actual edición del DLE (s.v.) se define falbalá como la "pieza casi cuadrada que se ponía en la abertura de un corte de la faldilla del cuarto trasero de la casaca".

Una representación del falbalá lo podemos apreciar en el final de la falda que lleva Carlota Joaquina de Borbón, la infanta de España (y futura reina de Portugal), en el retrato pintado por Ramón Bayeu hacia 1791 (figura 9).

23 Vid. nota 21.

24 También se define como las "cenéfas de cortinas puestas en la misma posicion" (NTLLE, 1726-1739, s.v. falbalâ). 


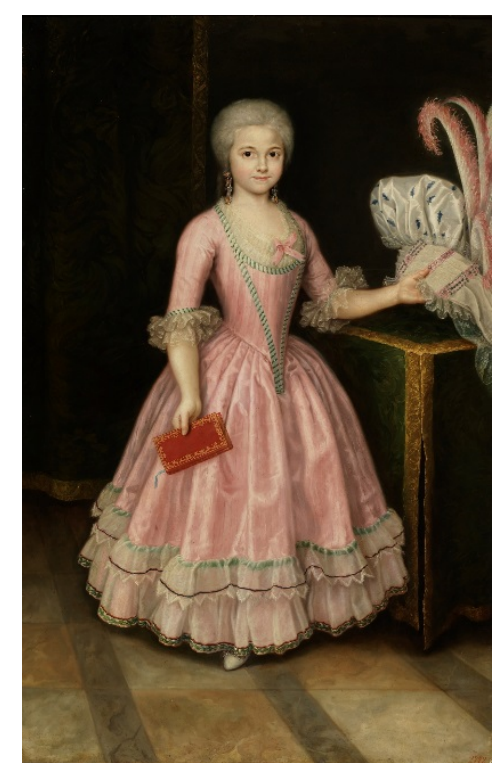

FIGURA 9. Ejemplo de falbalá en la falda de un vestido en el retrato de Carlota Joaquina de Borbón, infanta de España y futura reina de Portugal, de Ramón Bayeu (c. 1791).

\subsubsection{Palatina}

Por último, podemos hablar de la palatina "adorno de martas, seda, plumas, etc., que usaban las mujeres para cubrir y abrigar la garganta y el pecho a modo de una corbata ancha y tendida" (DLE, s.v.), atestiguado en dos inventarios aragoneses, uno del año 1739 (13) y otro del año 1764 (14):

(13) Más 8 onzas y tres quartas de dichos encajes de oro anchos y angostos para guarnizión de escusalí, paletina y petos que se entregaron a la bordadora (p. 2, línea 20$)^{25}$.

(14) Ocho paletinas de gassa y nuditos, negras, y tres de nuditos, las dos berdes y la otra azul (p. 69, línea 18) ${ }^{26}$.

Esta voz podría relacionarse con el francés palatine, tal y como apunta Terreros y Pando (1987 [1786-1793]: s.v. paletina), término que se remonta a un origen antroponímico, pues provendría "du nom de la princesse Palatine Anne de Gonzague (1616-1684) qui mit ce vêtement à la mode" (TLF , s.v. palatine $)^{27}$.

En cuanto a su cronología, en francés palatine se documenta desde las últimas décadas del siglo XVII (TLF , s.v. palatine). Sin embargo, en español, palatina aparece poco después, desde el Diccionario de Autoridades, y los ejemplos del CORDE que se

25 Vid. nota 21.

${ }^{26} \mathrm{Vid}$. nota 7.

27 Según el DLE (s.v. palatina), esta voz deriva de PALATīNUM. 
refieren a la definición propuesta son de mediados del siglo XVIII ${ }^{28}$. Por consiguiente, todo parece indicar que se trata de una palabra escasamente localizada en la documentación de fecha anterior al siglo XVIII, de acuerdo con los datos de los que se dispone hasta el momento.

La variante atestiguada en nuestros inventarios, paletina, resulta minoritaria como lema en los repertorios lexicográficos: de hecho, la Academia prefiere palatina desde el Diccionario de Autoridades (NTLLE, 1726-1739, s.v.) hasta la actual edición del DLE (s.v.), si bien se considera la forma paletina también en tres ediciones del Diccionario manual de 1950, 1980 y 1989 (NTLLE, 1950, 1980 y 1989, s.v.). Sin embargo, Terreros y Pando (1987 [1786-1793]: s.vv.), por su parte, en el caso de palatina, remite a paletina puesto que se decanta por la segunda como entrada propia en su vocabulario, definida como “adorno de la garganta que baja al pecho", a lo que añade que "en España las usan de muchas especies y materias: una especie hai en Francia á que llaman Tetonniere". La elección de Terreros y Pando por paletina va más en la línea de lo atestiguado en la documentación a la que se ha tenido acceso, pues tanto en las relaciones de bienes aragonesas como en los casos del CORDE se prefiere la variante con $e$ frente a la académica palatina.

Para ilustrar la voz palatina, remitimos al retrato de M. ${ }^{a}$ Isidra de Guzmán y de la Cerda, la llamada “doctora de Alcalá”, pintado por Joaquín de Inza hacia el año 1785, en el que M. ${ }^{a}$ Isidra luce una palatina de piel para proteger la zona del cuello y de los hombros (figura 10).

${ }^{28} \mathrm{El}$ primer ejemplo que figura en dicha base de datos es el siguiente: "JERÓNIMA: Compraré manteletas / de unas que he visto a la moda, / bata hecha de aguja toda, / paletinas y cofietas. / ANA: Cualquiera moda que salga, / por Dios, señora, que sean / las primeras que se vean / nosotras con ella" (N. Fernández de Moratín, La petimetra, 1762, CORDE). Otro caso con el sentido apuntado es tan solo un año posterior: "Y ¡qué bien / que le cae la cofia negra, / la paletina de lazos / y las hebillas de piedras!" (R. de la Cruz, El Alcalde Boca de verdades, 1763, CORDE). 


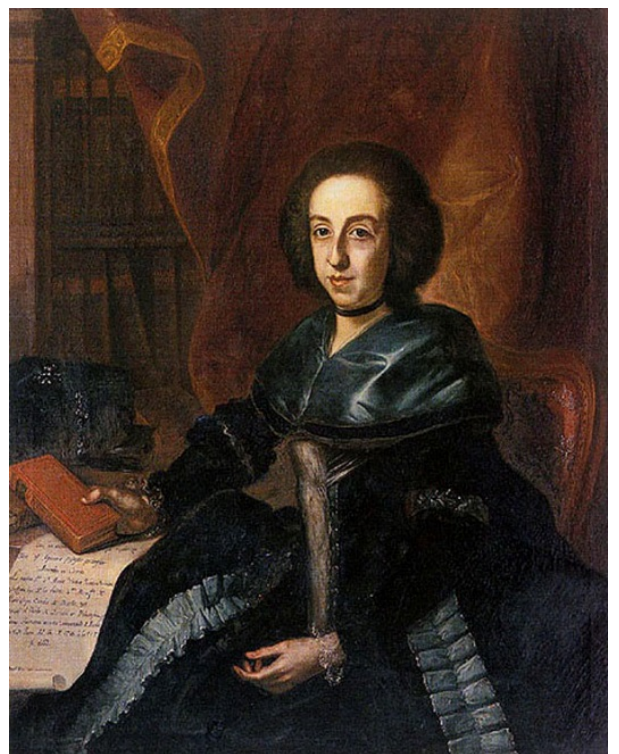

FIGURA 10. Ejemplo de palatina para hombros y cuello en el retrato de M. ${ }^{a}$ Isidra de Gurmán y de la Cerda, la llamada "doctora de Alcalá", de Joaquín Inza (c. 1785).

\section{A MODO DE CONCLUSIÓN}

Como hemos tratado de demostrar en este somero análisis de algunas voces escogidas presentes en inventarios de bienes aragoneses del siglo XVIII, este tipo de documentos resulta especialmente interesante para trazar o precisar el devenir de algunos términos, variantes o significados de la lengua española.

Así, más concretamente, y teniendo en cuenta como punto de partida la información encontrada en los textos analizados así como en los repertorios lexicográficos de la época dieciochesca, hemos visto que las mujeres adornaban sus cabelleras con airones, piochas y tembleques, tres términos que, aunque distintos, aludían a realidades próximas (diferentes tipos de tocados para el pelo). Aun con todo, hemos determinado que la voz piocha era más genérica que las restantes, puesto que, por una parte, se emplea de manera sinonímica para explicar airón en nuestra documentación y, por otra, se alude en nuestras relaciones de bienes a su naturaleza móvil, frente a lo que sucede en los ejemplos de tembleque.

En lo que respecta a las joyas, el empleo de aguacate con el sentido de piedra preciosa no gozaba de popularidad en el siglo XVIII a juzgar por la adición explicativa presente en nuestros inventarios. Asimismo, los pendientes podían ser de diversos tipos (arracadas y broquelillos) y, si bien en el Diccionario de Autoridades se hace referencia a que 
las primeras eran la parte colgante, en nuestros documentos se registra arracada a la manera tradicional, esto es, como un objeto de carácter independiente. Por otro lado, localizamos en estos manuscritos la variante manillera con el significado de manilla y nuevos ejemplos del reloj de repetición, invento reciente ideado en las últimas décadas del siglo XVII.

En cuanto a los accesorios, aunque los términos son de naturaleza diversa (el excusalí es un tipo de delantal; el falbalá, un aderezo del tontillo; y la palatina, un adorno protector de la zona de la garganta), tienen en común el hecho de tratarse de palabras escasamente documentadas en el siglo XVIII, a juzgar por la información manejada. Además, en nuestra documentación se da cuenta de su variabilidad formal, ya que en nuestros textos se prefieren las variantes escusalí, farbalá y paletina, respectivamente.

Por lo tanto, hemos visto cómo a través del estudio pormenorizado de los vocablos localizados en nuestras relaciones de bienes, se puede matizar el sentido de algunas palabras (airón, arracadas, piocha y tembleque), adelantar o aportar dataciones de dichas voces (falbalá, manillera, piocha, reloj de repetición y tembleque) y atestiguar formas alternantes en el tiempo (excusali $\sim$ escusalí, falbalá $\sim$ farbalá, manilla $\sim$ manillera y palatina $\sim$ paletina $).$ Y todo, claro está, con el fin de contribuir modestamente en el estudio histórico de nuestro bello idioma.

\section{BIBLIOGRAFÍA}

\section{Fuentes primarias}

Inventario de bienes y objetos encontrados en unas casas, sitas en la calle del Coso de Zaragoza, que pertenecieron a D. ${ }^{a}$ Prudenciana Portocarrero Funes de Villalpando, duquesa de Hijar. Fechado en Zaragoza, a 9 de junio de 1764. Consta de 75 páginas, sin numerar. Signatura: AHPZ, ADH, I-357-31/2.

Inventario de los bienes pertenecientes a la casa del Sr. Segovia. Fechado en Zaragoza, a 26 de marzo de 1733. Consta de 7 páginas, sin numerar. Signatura: AHPZ, J-302-1.

Inventario del menaje de la casa del conde de Aranda en Zaragoza. Fechado en Zaragoza, en noviembre de 1729. Consta de 13 páginas, sin numerar. Signatura: AHPZ, ADH, III-74-4.

Memoria de los vestidos de boda de D. ${ }^{a}$ Ana M. ${ }^{a}$ del Pilar Silva y Portocarrero, con sus hechuras y precios. Fechado en el año 1739. Consta de 6 páginas, sin numerar. Signatura: AHPZ, ADH, I-375-1/4.

Reparto de los bienes que quedaron por la muerte de Isabel López de Texeda, condesa de Morata, marquesa de Villaverde. Sin fecha. Consta de 9 páginas, sin numerar. Signatura: AHPZ, Argillo 131-6. 
Tasaciones de los bienes más valiosos de D. ${ }^{a}$ María Agustina Clavero y Dara. Incluye cinco tasaciones, fechadas en Zaragoza, a 24 de octubre, y a 2, 3 y 4 de noviembre de 1732 (la última no tiene fecha). Consta de 5 páginas, sin numerar. Signatura: AHPZ, J-302-1.

Fuentes secundarias

Arnal Purroy, M. (2000). Cambios grafemáticos en textos notariales aragoneses del siglo XVIII, a la luz de las reformas ortográficas de la RAE, Archivo de Filología Aragonesa, 56, pp. 117-136.

Benito, P. (2006). Bata del siglo XVIII, en VV.AA., Modelo del mes. Museo del Traje. Madrid: Museo del Traje, pp. 1-10 [en línea]: http://www.culturaydeporte.gob.es/mtraje/dam/jcr:00d863b7-5c614094-beda-aadb9e48c1d0/mdm05-2006.pdf [1/2/2019].

Bermejo, B. (2016). Vestido camisa y spencer (1800-1810). En VV.AA., Modelo del mes. Museo del Traje. Madrid: Museo del Traje, pp. 1-14 [en línea]: http://www.culturaydeporte.gob.es/mtraje/dam/jcr:cdbd4553-2b09-4e0d-81bf10abb14f4db5/03-2016.pdf [14/4/2019].

Calderón Campos, M. (2012). Variaciones gráficas y fonéticas del español del siglo XVIII en tres corpus

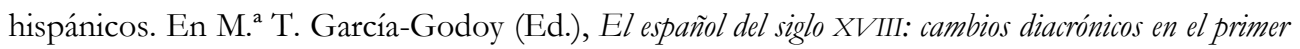
español moderno. Bern: Peter Lang, pp. 221-254.

Campos Souto, M. (2012). Voces gallegas (o no) en protocolos notariales del siglo XVIII: distrito de Carballo, Cuadernos del Instituto de Historia de la Lengua, 7, pp. 89-124.

CORDE $=$ Real Academia Española. Corpus diacrónico del español [en línea] $\leq$ http://www.rae.es $>$ [31/3/2019].

CorLexIn = Morala Rodríguez, J. R. (Dir.). Corpus léxico de inventarios [en línea] <http://web.frl.es/CORLEXIN.html> [31/3/2019].

DECH = Corominas, J. y J. A. Pascual (1980-1991). Diccionario crítico etimológico castellano e hispánico. 6 vols. Madrid: Gredos.

DHLE = Real Academia Española (1960-1996). Diccionario histórico de la lengua española [en línea] <http://web.frl.es/dh.html $>$ [31/3/2019].

DLE = Real Academia Española (2018). Diccionario de la lengua española. 23.2 edición [en línea] <http://www.rae.es $>[31 / 3 / 2019]$.

DUE $=$ Moliner, M. ${ }^{\mathrm{a}}$ (1966). Diccionario de uso del español. 2 vols. Madrid: Gredos.

Egido Fernández, M. C. (2008). Aspectos léxicos en documentación colonial del oriente boliviano (s. XVII-XVIII). En VV.AA., Actas del XV Congreso Internacional de la Asociación de Lingüística y Filología de la América Latina. Montevideo: ALFAL [publicación en CD].

Egido Fernández, M. C. (2012a). Léxico de la esclavitud en documentación americana: relaciones de bienes (siglos XVII-XVIII), Revista Internacional de Lingüistica Iberoamericana, X, 2 (20), pp. 27-47.

Egido Fernández, M. C. (2012b). Léxico y esclavitud en la América colonial (s. XVII-XVIII), Cuadernos del Instituto de Historia de la Lengua, 7, pp. 181-200.

Egido Fernández, M. C. (2012c). Léxico cotidiano en documentación colonial del Alto Perú (s. XVIIXVIII). En E. Montero Cartelle (ed.), Actas del VIII Congreso Internacional de Historia de la Lengua Española. Santiago de Compostela: Meubook, pp. 1299-1311. 
Egido Fernández, M. C. (2013). Indigenismos en la vida cotidiana de la América colonial (s. XVII-XVIII), Signo y Seña, 23, pp. 23-38.

García Navarro, J. (2006). Zapatos y medias del siglo XVIII. En VV.AA., Modelo del mes. Museo del Traje. Madrid: Museo del Traje, pp. 1-12 [en línea]: http://www.culturaydeporte.gob.es/mtraje/dam/jcr:4f6b440c-73e5-41bf-90b79b90ccfdad18/mdm06-2006.pdf [12/12/2018].

Gómez del Val, R. (2008). Traje de maja. En VV. AA., Modelo del mes. Museo del Traje. Madrid: Museo del Traje, pp. 1-10 [en línea]: http://www.culturaydeporte.gob.es/mtraje/dam/jcr:9455733a-e98d4428-a2e9-1d6f22201c84/04-2008.pdf [4/5/2019].

Gómez Seibane, S. y J. L. Ramírez Luengo (2007). El castellano de Bilbao en el siglo XVIII. Materiales para su estudio. Bilbao: Universidad de Deusto.

Imízcoz Beunza, J. M. (1996). Los inventarios de bienes alaveses en los siglos XVI, XVII y XVIII. Estructura, contenidos y evolución, en M. R. Porres Mariajuán (dir.), Aproximación metodológica a los protocolos notariales de Álava (Edad Moderna). Bilbao: Universidad del País Vasco, pp. 145-186.

Landes, D. S. (2007). Revolución en el tiempo: el relojy la formación del mundo moderno. Barcelona: Crítica.

Leira Sánchez, A. (2007). La moda en España durante el siglo XVIII, Indumenta: Revista del Museo del Traje, 0, pp. 87-94 [en línea]: http://www.culturaydeporte.gob.es/mtraje/dam/jcr:2b32d6d8-20cd4331-b94a-e0b1bc21524f/indumenta00-09-als.pdf [11/11/2018].

Leira Sánchez, A. (2008). Vestido hecho a la inglesa. En VV.AA., Modelo del mes. Museo del Traje. Madrid: Museo del Traje, pp. 1-15 [en línea]: http://www.culturaydeporte.gob.es/mtraje/dam/jcr:a430617c-f9f6-438d-b1d9695cafa2dd99/12-2008.pdf [23/11/2018].

NTLLE = Real Academia Española. Nuevo tesoro lexicográfico de la lengua española [en línea] $\leq$ http://buscon.rae.es/ntlle/SrvltGUILoginNtlle $>$ [31/3/2019].

Ortiz Cruz, D. (2010). Inventarios aragoneses de la Casa de Aranda en el siglo XVIII: estudio gráfico, Res Diachronicae, 8, pp. 71-92.

Ortiz Cruz, D. (2015a). Los inventarios de bienes en el norte peninsular: el caso de un inventario de un mercader zaragozano (1748), Res Diacbronicae, 13, pp. 49-57.

Ortiz Cruz, D. (2015b). Aproximación al léxico relativo a los molinos localizado en inventarios de bienes dieciochescos del Archivo Ducal de Híjar, Rujiar: miscelánea del Centro de Estudios Bajo Martín, 16, pp. 177-199.

Ortiz Cruz, D. (2017a). Cuestiones problemáticas de las denominaciones textiles a través de inventarios de bienes aragoneses de los siglos XVII y XVIII. En J. González Gómez, V. Lara Bermejo y O. León Zurdo (Eds.), Tenera Experientia. Miradas jóvenes a la Historiografia y la Historia de la Lengua Española. Madrid: Universidad Autónoma de Madrid, pp. 207-216.

Ortiz Cruz, D. (2017b). Cuestiones problemáticas de las denominaciones textiles a través de inventarios de bienes aragoneses de los siglos XVII y XVIII (II): el caso de las sedas, Res Diacbronicae, 14 (2), pp. 37-45.

Ortiz Cruz, D. (2018). Tendencias ortográficas en inventarios de bienes aragoneses del siglo XVIII, Archivo de Filología Aragonesa, 74, pp. 99-137. 
Ortiz Cruz, D. (en prensa). Estudio léxico-semántico de inventarios de bienes aragoneses del siglo XVIII. Zaragoza: Institución "Fernando el Católico".

Puche Lorenzo, M. Á. (2009a). Ropas y flores en el siglo XVIII a través del léxico. En J. F. Lorenzo Rojas, M. J. Sánchez Rodríguez y E. R. Montoro Cano (Coords.), Lengua e historia social: la importancia de la moda. Granada: Universidad de Granada, pp. 129-146.

Puche Lorenzo, M. Á. (2009b). Usos, costumbres e influencias de otras tierras en el mobiliario y las ropas del siglo XVIII a través del léxico. En VV.AA., Actas del Congreso Internacional "Imagen y Apariencia”. Murcia: Universidad de Murcia, pp. 1-14 [en línea]: <http://congresos.um.es/imagenyapariencia/imagenyapariencia2008/paper/viewFile/2711/2 $\underline{651 \geq[14 / 10 / 2018 .]}$

Redondo Solace, M. (2007). Polonesa del siglo XVIII. En VV.AA., Modelo del mes. Museo del Traje. Madrid: Museo del Traje, pp. $1-14$ [en línea]: http://www.culturaydeporte.gob.es/mtraje/dam/jcr:7cda2f17-20fd-4d53-bbc92b5fcc073079/06-2007.pdf [3/3/2019].

Santos Llorite, M. R. (2001). Los inventarios de bienes como fuente para la Historia de la Ciencia y de la Técnica: Zaragoza (1550-1600). En A. Ubieto (Ed.), III Jornadas de Estudios sobre Aragón en el umbral del siglo XXI, Caspe, 15-17 de diciembre de 2000. Zaragoza: Instituto de Ciencias de la Educación, pp. 1211-1221.

Terreros y Pando, E. (1987 [1786-1793]). Diccionario castellano con las voces de ciencias y artes. 4 vols. Madrid: Arco/Libros.

$\mathrm{TLF}_{\mathrm{I}}=$ Trésor de la langue française informatisé [en línea] <http://atilf.atilf.fr/tlf.htm $>$ [31/3/2019].

Vivancos Mulero, M. E. (2012). El sufijo -ico/-iquio como caracterizador dialectal del español murciano (siglo XVIII). En M. ${ }^{a}$ T. García-Godoy (Ed.), El español del siglo XVIII: cambios diacrónicos en el primer español moderno. Bern: Peter Lang, pp. 313-332. 